\section{A retrospective, practice-based, clinical evaluation of Fuji IX restorations aged over five years placed in load-bearing cavities}

\author{
F. J. T. Burke ${ }^{* 1}$ and J. S. Bardha
}

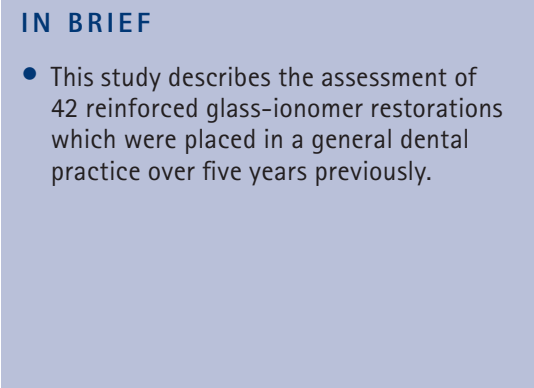

Objective To evaluate reinforced glass-ionomer restorations which had been placed in a general dental practice more than five years previously. Method Patients who were identified as having received reinforced one or more reinforced glassionomer restorations were invited to attend for an examination of their restorations using scientific evaluation criteria, by one independent examiner and the dentist who owned the practice. Results Forty-two restorations were assessed, their mean age being 7 years and 9 months, in patients of mean age 57 years: 86\% achieved an A rating for anatomic form, 69\% A for marginal integrity, 81\% A for surface roughness and 2\% A for colour match. Conclusions The restorations which were assessed were found to be performing satisfactorily at periods of over five years. However, the proportion of the total number of reinforced glass-ionomer restorations placed in the participating dental practice which this represents is not known.

\section{INTRODUCTION}

\section{Practice-based research}

The majority of research into the effectiveness of dental materials is carried out in dental hospitals or other academic institutions rather than in general dental practice, although general practice is where the majority of dental treatment is performed worldwide. Reasons for this divergence include the potential cost, given that practices are geared to the efficient treatment of patients rather than research. The training of general practitioners in research methods may also be incomplete. However, there are many reasons why dental practice increasingly should become the prime location for clinical dental research. ${ }^{1}$ Dental practice is the real world, with a wide variety of patients, and clinicians with differing undergraduate and postgraduate backgrounds. Practice-based research could be considered to assess effectiveness (that is, how something performs in the

\footnotetext{
Primary Dental Care Research Unit, University of Birmingham, School of Dentistry, St. Chad's Queensway, Birmingham, B4 6NN, UK.

${ }^{*}$ Correspondence to: Professor Trevor Burke Tel: 0121237 2767; Fax: 01212372768 Email:f.j.t.burke@bham.ac.uk
}

Online article number E9

Refereed Paper - accepted 14 June 2013

DOI: $10.1038 /$ sj.bdj.2013.880

${ }^{\circ}$ British Dental Journal 2013; 215: E9 real world), whereas, assessment of restorations in a hospital or academic environment where the patients are subject to rigorous inclusion/exclusion criteria could be considered to represent efficacy (how something performs in an ideal environment). Accordingly, if a technique or material is to be successful, it must be readily operated in the dental practice situation.

A number of types of research may be considered particularly appropriate to dental practice. These include clinical trials of materials and techniques, assessment of treatment trends, and assessment of dentists' behaviour and attitudes. For the practitioner, there is the benefit of being involved in something not normally within the daily routine of practice. Patients have also been found to approve of practitioner involvement in research, with the practice and practitioner's professional image being enhanced. ${ }^{2}$ There is increasing evidence that practitioners wish to become involved in research in their practices, both in the UK and in the US, given the success of UK-based practice-based research groups such as the PREP (Product Research and Evaluation by Practitioners) Panel, which has recently celebrated its 20th anniversary, ${ }^{3}$ and BRIDGE (Birmingham Research in Dental General practicE) ${ }^{4}$, plus the establishment of a number of practice research networks in the US. ${ }^{5}$

\section{Glass-ionomer materials}

Glass-ionomer cements were developed in the early 1970s. These materials comprised a fluoro-alumino-silicate (FAS) glass mixed with a polyacrylic acid. ${ }^{6}$ Their popularity increased through the 1980s, and in 2000 these materials were used in the placement of circa 1.7 million restorations in the NHS in England and Wales, mainly in Class V non-load-bearing cavities. ${ }^{7}$ Principal advantages of glass-ionomer materials include their good compressive strength, their reliable adhesion to tooth substance (which, in turn, reduces the need for the clinician to cut sound tooth substance to create retention for the restoration), and release of fluoride, which may inhibit the progress of caries around the restoration (although the literature on this is by no means equivocal). ${ }^{9}$ Disadvantages of conventional materials included poor tensile/flexural strengths and suboptimal wear resistance, which precluded the use of these materials in load-bearing cavities, moisture sensitivity, and poor aesthetics, because of their opacity. ${ }^{8}$

The most recently developed generation of glass-ionomer materials have been termed fast-setting, high-strength, or reinforced glass ionomers. This group includes Fuji IX (GC, Tokyo, Japan), Chemflex (Dentsply, Weybridge, UK), and Ketac-Molar Easymix (3M ESPE, Seefeld, 
Germany). Manufacturers claim improved early physical properties and resistance to dissolution over conventional glass ionomers ${ }^{10}$ this improvement being due to a reduction in the size of the glass particles in the matrix allowing a faster speed of reaction between the glass and the polyacrylic acid. These materials are stiffer when mixed and have been termed 'packable' as a result. Manufacturers have considered that a reinforced glass-ionomer material may be suitable as long-term temporary restoration of Class I and II cavities in permanent teeth (Chemflex), or permanent small Class I restorations, ${ }^{11}$ notwithstanding its suggested use in Class III and V cavities, Class I and II cavities in primary teeth, fissure fillings, core build-ups and atraumatic restorative treatment (ART) technique. $^{9}$ The manufacturers of Fuji IX GP, however, suggest that this material is suitable for Class I, II and V restorations in permanent and primary teeth. ${ }^{12}$

General dental practitioners are constantly faced with the requests of their patients, and these may include toothcoloured restorations in posterior teeth and low cost. It is therefore not surprising that anecdotal information became available that a number of UK general dental practitioners were using reinforced glass ionomers to restore cavities in loadbearing situations in posterior teeth. These may be placed in bulk, resulting in a saving in time when compared with the time-consuming incremental build-up required for posterior composite restorations. This, in turn, results in a restoration which is less expensive than the equivalent posterior composite.

The authors became aware of three dental practitioners who either placed reinforced glass-ionomer restorations in posterior teeth, or who had purchased dental practices in which their predecessor had placed such restorations. Accordingly, a practice-based retrospective clinical evaluation of 169 Fuji IX restorations was carried out, with the results, which indicated high rates of success, being published in the British Dental Journal in 2007. ${ }^{13}$ The three practitioners involved in that project were contacted in early 2007 and asked if they would be prepared to extend the project to five years. All three responded, but one had retired from practice, and a second had relocated and was uncertain as to whether he could identify the patients involved in the original project. The third practitioner agreed to participate in a retrospective evaluation.

\section{STUDY OBJECTIVES}

The purpose of this practice-based clinical trial was therefore to carry out a retrospective evaluation of Fuji IX (GC Europe, Leuven, Belgium) restorations placed in Class I and II cavities in patients who attended one Birmingham-based dental practice. The study focused on Fuji IX restorations placed before 2001 by the previous owner of the practice, and those placed by the present owner after 2001, with the restorations being assessed, using USPHS criteria ${ }^{11}$ by the present practice owner and by an examiner who was trained and calibrated in the assessment of restorations.

\section{STUDY DESIGN}

Ethical approval was obtained for the study from the West Midlands Research Ethics Committee.

The study was a practice-based, retrospective clinical evaluation of load-bearing restorations formed in Fuji IX. The study examined restorations at five or more years' post placement, with patients being invited to attend a special appointment for assessment of their restorations.

\section{Objectives of clinical investigation}

To evaluate the clinical performance of Fuji IX restorations in Class I and II cavities using USPHS criteria (Table 1). Primary end points:

- Retention of the restoration

- Lack of fracture of the restoration

- Margin integrity

- Secondary caries status

- Post-operative sensitivity secondary end point:

- Surface quality

\section{Patient population}

Subjects involved in this investigation were those who had received one or more Fuji IX restorations and who were prepared to attend for an independent clinical examination of their restoration(s). Patients were remunerated £20 for attending for examination.

\section{Inclusion and exclusion criteria}

There were no specific inclusion and exclusion criteria. However, it is suggested that general inclusion criteria for prescribing a Fuji IX restoration in a load-bearing situation in a posterior tooth include:

- patients with a molar supported permanent dentition free of any clinically significant occlusal interferences

- patients whose dentitions were well maintained and free of any active, untreated periodontal disease.

Patients who would generally be excluded from receiving Fuji IX restorations include:

- a history of any adverse reaction to clinical materials of the type to be used in the study

- evidence of occlusal parafunction and/ or pathological tooth wear

- medical and/or dental histories which could possibly complicate the provision of the proposed restoration and/or influence the behaviour and performance of the restorations in clinical service

- irregular dental attenders.

\section{Recalls}

Patients who, when attending for routine examination, were found (following the examination of their clinical notes) to have received one or more Fuji IX load-bearing restorations in a posterior tooth five or more years previously, were invited to participate in the study. They were presented with a patient information leaflet and were given two weeks to decide whether they wished to participate.

Those willing to take part were given a further appointment for the review of their restorations.

\section{Review procedure}

All restorations were inspected and assessed according to the codes and criteria set out in Table 1, which are based on the criteria laid down by Ryge,,${ }^{14}$ with these assessments being undertaken by one trained and calibrated examiner (FJTB) with the assistance of the clinician (JB), in whose dental practice the restorations had been placed.

The assessment included: evaluation of anatomic form, margin adaptation, surface roughness, colour match and presence or absence of secondary caries. 
Table 1 Criteria for restoration evaluation ( ${ }^{*}=$ unacceptable), adapted from reference 11

\section{Restoration retention}

A. Restoration completely present

B. Restoration partly lost, but not in need of replacement

$C^{*}$. Restoration lost, in need of immediate replacement

Anatomic form

A. Restoration is contiguous with existing anatomic form

B. Restoration is under-contoured but no dentine or base exposed

$C^{*}$. Sufficient restorative material is missing so that dentine or base is exposed

Margin integrity

A. No visible evidence of a crevice along the margin into which a probe will catch

B. Probe catches in a crevice along the margin, no dentine or base exposed

$\mathrm{C}^{*}$. Visible evidence of a crevice with exposure of dentine or base

\section{Margin discolouration}

A. No discolouration evident at margin

B. Slight staining at margin

$C^{*}$. Obvious staining, cannot be polished away

Colour match

A Restoration matches adjacent tooth structure in colour and translucency

B. Mismatch in colour and translucency but within an acceptable range

$C^{*}$. Mismatch in colour and translucency outside acceptable range

Surface roughness

A. Smooth surface with no irritation of adjacent tissues

B. Dull, matte surface, can be refinished

$C^{*}$. Shallow surface pitting is present. Rough, cannot be polished

\section{Staining}

A. No staining of restoration

B. Staining that can be polished off

$C^{*}$. Staining that cannot be polished off.

\section{Secondary caries}

A. Restoration is judged caries free.

$B^{*}$. Secondary caries detected

When decisions of assessors differed, a consensus opinion was agreed before the patient was dismissed. In the event of a restoration being unsatisfactory, details of the mode of failure was recorded and arrangements made for the necessary remedial work to be carried out.

\section{RESULTS}

- A total of 42 restorations, in 25 patients (14 male, 11 female), were available for assessment

- The mean age of the restorations was
7 years and 9 months (range 5 to 10 years), while the mean age of the patients was 57 years (range 33 to 81 years)

- The greatest number of restorations examined in one patient was three

- One of the teeth examined was mobile, this having an occluso-buccal restoration in 47 in a 78-year-old patient

- The distribution of the restorations is presented in Table 2, with 13 restorations being found to be in premolar teeth and 29 in molar teeth
Table 2 Distribution of restoration types

\begin{tabular}{l|l} 
Type of restoration & Number examined \\
\hline Class I & 7 \\
\hline Class I with extension & 6 \\
\hline Class II & 9 \\
\hline Class II with extension & 4 \\
\hline $\begin{array}{l}\text { Class II, pin retained, } \\
\text { with extension }\end{array}$ & 1 \\
\hline Mesio-occlusal-distal & 11 \\
\hline MOD with cusp replacement & 4
\end{tabular}

The restorations were assessed using a modification of USPHS (Ryge) criteria as per Table 1

- All but one of the original restorations placed in the selected patients were available for examination. This restoration, a Class I, had failed at 5 years and had been replaced with another Fuji IX restoration which was satisfactory

- Regarding anatomic form, 85.7\% ( $\mathrm{n}=36$ ) of restorations were assessed as $\mathrm{A}$, the remainder being assessed as B. No restorations scored an unsatisfactory $\mathrm{C}$ grade

- Regarding surface roughness, 81\% ( $n=34)$ were assessed as A, 17\% ( $n=7)$ being assessed as B and one as $\mathrm{C}$

- Regarding surface staining, 95.2\% ( $\mathrm{n}=40$ ) were assessed as A, the remainder as B

- Regarding marginal integrity, 69.0\% ( $\mathrm{n}=29$ ) were assessed as A, 28.6\% ( $\mathrm{n}=12)$ as B and $2.4 \%(\mathrm{n}=1)$ as $C$

- Regarding marginal discolouration, 64.3\% ( $\mathrm{n}=27$ ) were assessed as A and $35.7 \%(\mathrm{n}=15)$ as B

- Regarding colour match, 2.4\% ( $\mathrm{n}=1)$ was assessed as $\mathrm{A}$ and the remainder $(97.6 \% ; n=41)$ as $\mathrm{B}$.

\section{DISCUSSION}

The present study presents 'real world' data from general dental practice and the restorations assessed in the study were therefore placed within the time pressures pertaining to a busy UK dental practice. In the initial study which catalysed the present work, ${ }^{13}$ the participating practitioners were given training in the use of USPHS criteria and assessed the restorations themselves. It could, however, be argued that these practitioners might not make a totally objective 
assessment of the restorations which they had placed. This procedure was not therefore considered to be scientifically robust, so the present study sought to improve the robustness of the work and therefore employed an independent observer. This necessarily meant that: (i) patients had to attend at pre-arranged times for the assessment of their restorations, (ii) that ethical committee approval was needed, and (iii) that the patients had to be reimbursed for their attendance, notwithstanding the cost of sending the observer to the practice.

In the participating practice, the predecessor to the practitioner who assisted with the assessment placed the Fuji IX restorations which had been placed before 2001, while the restorations placed after 2001 were placed by the owner of the practice who also assisted with the assessments. The presence of an independent trained and calibrated examiner in the present study could, however, be considered to provide a more robust assessment of the restorations. Notwithstanding this, however, the sample size is small and a larger sample size would have given the study more validity.

Patients who were identified as having received Fuji IX restorations in load-bearing surfaces of their posterior teeth were invited to participate in the study. However, the proportion of the total number of Fuji IX restorations that this represents in this particular practice is not known, this being a potential limitation of the study. What the present work appears to indicate is that, under certain circumstances, Fuji IX restorations may provide good mediumto long-term service. What these circumstances are is not clear from the data that were collected. However, they might include low to normal levels of occlusal force and low levels of acidogenic plaque, as this has been shown to cause caries-like dissolution of glass-ionomer restorations at contact areas. ${ }^{15}$ The absence of a contact area might also, therefore, be a factor in optimising survival of the restorations. Notwithstanding this, however, circa $80 \%$ of the Class II restorations assessed in the present work did have a contact with the adjacent tooth.

Disadvantages of conventional glassionomer materials included poor tensile and flexural strengths, ${ }^{6}$ which, in effect, precluded the use of these materials in load-bearing cavities, with a failure rate twice that of amalgam being noted even in the less demanding conditions of restorations in primary teeth. ${ }^{16,17}$ Qvist and colleagues, reporting the eight-year results of RMGI restorations in primary teeth, considered that these materials should be preferred to conventional glass-ionomer materials. ${ }^{18}$ However, there is a paucity of data on the performance of any type of glass ionomer in load-bearing situations in posterior teeth in adult patients. The present study, while lacking the scientific rigour of controlled prospective evaluations, does appear to suggest that a reinforced glass-ionomer, Fuji IX, may perform satisfactorily in periods of over 5 years in Class I and II cavities, some of which were extensive cusp replacement restorations (Table 2). However, these results suggest a need for a properly controlled long-term prospective studies and to quantify the cariostatic effect of glass-ionomer restorations placed in load-bearing cavities. In this regard, the results of a study by Basso and Heiss on Equia Fil (GC, Leuven, Belgium), a material derived from the material assessed in the present study, have recently been published in abstract form. ${ }^{19}$ These workers evaluated 319 restorations (of 380 which were originally placed: 83 Class I, 184 Class II, 72 Class V) at a mean follow up time of 40 months, with 22 restorations having chipped margins and 14 restorations lost or damaged, a general success rate of $95.6 \%$, and with a higher failure rate in Class II restorations. The authors concluded that the restorative system used appeared to be a reliable choice for permanent dental restorations, even in the surfaces of molars and premolars.

Results of one in vitro study have suggested that Fuji IX has a wear resistance not dissimilar to a resin composite. ${ }^{20}$ Another study has suggested that the early wear resistance of highly-viscous glass ionomers should be improved, ${ }^{21}$ but also considered that these materials may compete with composites as far as long-term wear is concerned. The results of the present study suggest that wear of the occlusal surfaces of the restorations assessed was not a problem for the majority of the restorations during the period of this assessment, as there was little evidence of that. Figures 1, 2 and 3 present three typical restorations. There may be

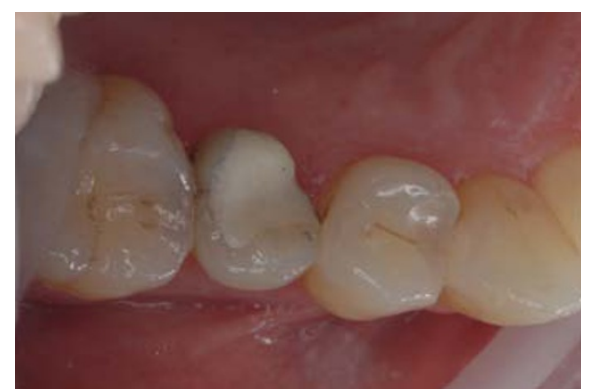

Fig. 1 A mesio-occlusal cusp replacement restoration in a maxillary premolar tooth at six years

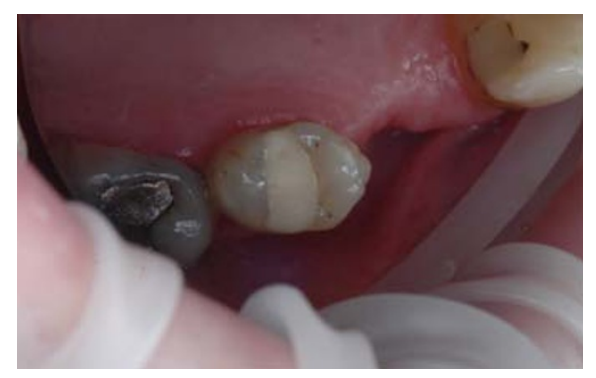

Fig. 2 MOD restoration in a rotated maxillary premolar tooth at six years

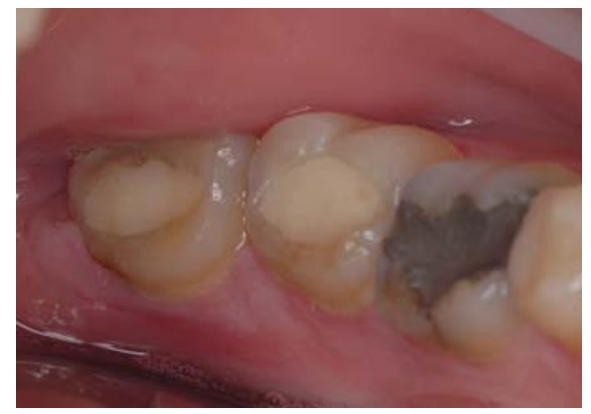

Fig. 3 Class I restorations in mandibular molar teeth at nine years in a 42-year-old male patient

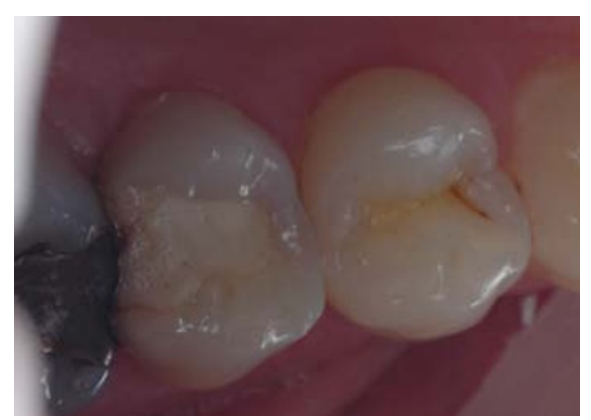

Fig. 4 Unacceptable pitting in a premolar restoration at six years

some 'flattening' of the restorations, but, in the absence of baseline illustrations, it is not possible to judge whether the surface contour had been lost. Regarding surface roughness, one restoration scored C (unsatisfactory) but no dietary or other influences were identified for this patient (Fig. 4). 
Results from an overview of ten crosssectional studies, which included 2,137 glass-ionomer restorations, have indicated that secondary caries is the reason for failure of between $17 \%$ and $40 \%$ of glass-ionomer restorations. ${ }^{22}$ Additionally, the benefit of fluoride release from glassionomer restorations in respect of cariostasis is not clear. ${ }^{9}$ However, the results of the present study suggest that secondary caries may not be problem associated with reinforced glass-ionomer restorations at periods of more than five years. In this regard, in his 2011 review of systematic reviews, Mickenautsch ${ }^{23}$ considered that Fuji IX and KetacMolar (3M ESPE, Seefeld, Germany) had a higher caries-preventive effect than that of amalgam, so the results in the present study may not be considered a surprise.

The colour match was not ideal in all but one restoration, not surprising given the generally-accepted suboptimal appearance and opacity of glass-ionomer materials. However, this did not appear to present any difficulties when patients were asked regarding the appearance of their restorations. No problems with collection of surface stain were identified. Marginal discolouration was noted in circa one third of restorations, but the absence of secondary caries generally throughout the study could be considered to indicate that the marginal discolouration was not indicative of early caries activity.

Worldwide, there appears to be an increasing demand for tooth-coloured restorations in posterior teeth. ${ }^{24}$ However, although the clinical performance of resin composite restorations in posterior teeth has been shown to be similar to that of amalgam restorations, ${ }^{25}$ restorations in posterior composite may not be considered as cost-effective as amalgam because they have been considered to take 2.5 times longer to place than equivalent amalgam restorations..$^{26}$ It could therefore be considered unsurprising, especially in these times of economic downturn, if patients and their dental healthcare workers, were not to investigate the use of a tooth-coloured, easily-handled material, such as glassionomer, as a replacement for amalgam. The principal cost in any restoration is the clinicians' time, so a material which can be placed quickly could be considered attractive in this respect. The results of this study indicate that, in clinical situations where there are no adverse situations at work (such as high occlusal loading or an acidogenic plaque ${ }^{15}$ ), certain restorations in Fuji IX may provide reasonable longevity.

\section{CONCLUSION}

Within the limitations of the study, selected reinforced glass-ionomer restorations placed in load-bearing situations, in patients attending one dental practice in the UK, were found to be performing satisfactorily at periods of over five years. Further, controlled, prospective investigations are indicated to more fully assess the performance of such restorations.

The authors wish to thank GC Europe, for the partial funding of this project and the patients who attended to have their restorations assessed.

1. Mandel I D. Clinical research - the silent partner in dental practice. Quintessence Int 1993; 24: 453-463.

2. Burke F J T, McCord J F. Research in dental practice - problems and solutions. Br Dent J 1993; 175: 396-398.

3. Burke F J T, Crisp R J. Twenty years of handling evaluations and practice-based research by the PREP Panel. Dent Update 2013; 40: 339-342.

4. Stewardson D A, Creanor S, Thornley P et al. The survival of Class $V$ restorations in general dental practice: Part 3, five year survival. Br Dent J 2012 212: E14.

5. Gordan V V. Translating research into everyday clinical practice: Lessons learned from a USA dental practice-based research network. Dent Mater 2013; 29: 3-9.

6. Wilson A D, Kent B E. A new translucent cement for dentistry. The glass-ionomer cement. Br Dent J
1972; 132: 133-135

7. Dental Practice Board. Digest of statistics, April 2002 - March 2003. Eastbourne: Dental Practice Board UK, 2003.

8. Combe E C, Burke F J T, Douglas W H. Clinical Dental Materials. Kluwer Academic Publishers, 1999.

9. Randall R C. Wilson N H F. Glass ionomer restoratives: A systematic review of a secondary caries treatment effect. J Dent Res 1999; 78: 628-637.

10. Dentsply De Trey GmbH professional research Chemflex Technical Manual. p 25. Germany: Dentsply De Trey, 1998

11. Ketac Molar Quick product brochure. Online information available at www.3MESPE.com (accessed July 2013)

12. GC Fuji I X, Fuji IX GP fast manual. Online information available at www.gceurope.com (accessed July 2013).

13. Burke F J T, Siddons C, Phipps S, Bardha J, Crisp R J, Dopheide B. Clinical performance of reinforced glass-ionomer restorations placed in UK dental practices. Br Dent J 2007; 203: E2. Research summary: Br Dent J 2007; 203: 529-530.

14. Ryge G. Clinical criteria. Int Dent J 1980; 30: 347-357.

15. Scholtanus J D, Huysmans M C. Clinical failure of a highly viscous glass-ionomer material over a six year period: A retrospective study. J Dent 2007; 35: 156-162.

16. Welbury R R, Walls A W G, Murray J J, McCabe J F. The 5-year results of a clinical trial comparing a glass polyalkenoate (ionomer) cement restoration with an amalgam restoration. Br Dent J 1991; 170: 177-181.

17. Ostlund J, Moller K, Koch Amalgam, G. Composite resin and glass-ionomer cement in class II restorations in primary molars - a three yea clinical evaluation. Swed Dent J 1992; 16: 81-86

18. Qvist V, Manscher E, Teglers P T. Resin-modified and conventional glass-ionomer restorations in primary teeth: 8-year results. J Dent 2004; 32: 285-294.

19. Basso M, Heiss M A. Permanent restorations with glass-ionomer cements: clinical evaluation on 319 cases. J Dent Res 2013; 92: Special Issue A. Abstract 594.

20. Yap A U J, Teo J C M, Teoh S H. Comparative wear resistance of reinforced glass-ionomer restorative materials. Oper Dent 2001; 26: 343-348.

21. Van Duinen R N B, Kleverlaan CJ, de Gee A J, Werner A, Felizer A J. Early and long-term wear of "Fastset" conventional glass-ionomer cements. Dent Mater 2005; 21 : 716-720.

22. Deligeorgi V, Mjor I A, Wilson N H F. An overview of reasons for the placement and replacement of restorations. Primary Dent Care 2001: 8: 5-11.

23. Mickenautsch S. How well are GIC product labels related to current systematic review research? Dent Update 2011; 38: 634-644.

24. Burke F J T. Amalgam to tooth-coloured materials - implications for clinical practice and dental education: governmental restrictions and amalgamusage survey results. J Dent 2004; 32: 343-350.

25. Manhart J, Chen H Y, Hamm G, Hickel R. Review of the clinical survival of direct and indirect restorations in posterior teeth of the permanent dentition. Oper Dent 2004; 29: 481-508.

26. Burke F J T. Attitudes to posterior composite filling materials: A survey of 80 patients. Dent Update 1989; 16: 114-120. 